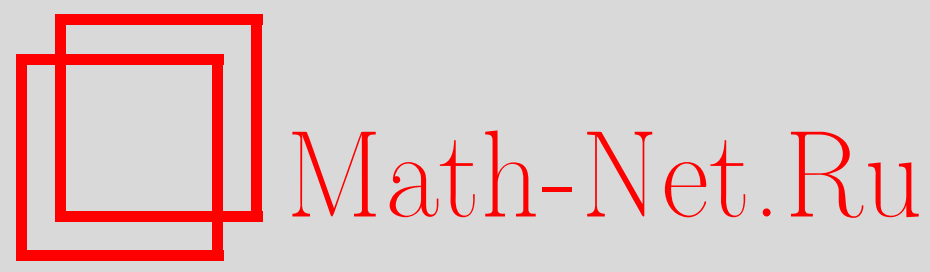

С. В. Асташкин, О дизъюнктной строгой сингулярности вложений симметричных пространств, Матем. заметки, 1999, том 65, выпуск 1, 3-14

DOI: https://doi.org/10.4213/mzm1022

Использование Общероссийского математического портала Math-Net.Ru подразумевает, что вы прочитали и согласны с пользовательским соглашением http://www . mathnet.ru/rus/agreement

Параметры загрузки:

IP : 54.166.219.16

26 апреля 2023 г., 15:56:24

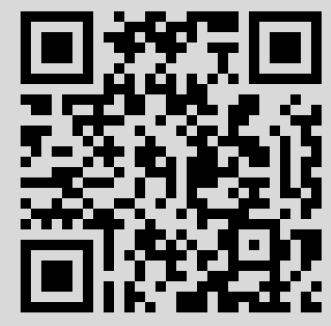




\section{О ДИЗЪЮНКТНОЙ СТРОГОЙ СИНГУЛЯРНОСТИ ВЛОЖЕНИЙ СИММЕТРИЧНЫХ ПРОСТРАНСТВ}

\section{С. В. Асташкин}

В работе рассматриваются вопросы, связанные с дизъюнктной строгой сингулярностью вложений симметричных пространств функций, определенных на отрезке. Найдено условие, использующее фундаментальные функции пространств, которое обеспечивает наличие "зазора" между ними, достаточного для того, чтобы вложение одного пространства в другое обладало этим свойством. Показано, что это условие в определенном смысле точное. Попутно получены необходимые и достаточные условия для того, чтобы дизъюнктно строго сингулярным было вложение одного пространства Лоренца в другое пространство Лоренца (и аналогичное утверждение для пространств Марцинкевича). Доказаны также и некоторые другие результаты.

Библиография: 12 названий.

Введение. Напомним, что линейньй оператор $T$, ограниченньй из банахова пространства $X$ в банахово пространство $Y$, назьвают строго сингулярнылм (или оператором Kamo), если в $X$ не существует бесконечномерного подпространства $Z$ такого, что сужение $T$ на $Z$ является изоморфизмом.

Класс строго сингулярных операторов в течение последних десятилетий интенсивно изучался (библиографию см., например, в монографии [1]). Среди исторически первых и важных для дальнейшего изложения результатов отметим теорему Гротендика о том, что строго сингулярньп является оператор тождественного вложения пространства $L_{\infty}(\Omega, \mu)$ в $L_{p}(\Omega, \mu)(1 \leqslant p<\infty)$, где $\mu$ - вероятностная мера на пространстве $\Omega$ (см. [2] или [3, с. 133]). Однако, как правило, вложения симметричных пространств (определение см. ниже) этим свойством не обладают. Причина - существование "сквозных" подпространств (например, подпространства, порожденного системой функций Радемахера [4]). Отчасти и в связи с этим обстоятельством в 1989 году в работе [5] было введено близкое понятие дизъюнктно строго сингулярного оператора.

Линейньй оператор $T$, ограниченный из банаховой решетки $X$ в банахово пространство $Y$, назьвается дизбюнктно строго сингулярным (или имеет DSS-свойство), если не существует последовательности ненулевых дизъюнктных векторов $\left\{x_{n}\right\}_{n=1}^{\infty}$ в $X$, для которых сужение $T$ на замкнутую линейную оболочку $\left[x_{n}\right]$ является изоморфизмом.

Ясно, что любой строго сингулярный оператор является DSS-оператором. Как показывает простой пример, обратное неверно. Так, оператор тождественного вложения $I: L_{p}[0,1] \rightarrow L_{q}[0,1](1 \leqslant q<p \leqslant \infty)$ имеет DSS-свойство, так как для дизъюнктных функций $x_{n} \in L_{r}[0,1](1 \leqslant r \leqslant \infty)$ замкнутая линейная оболочка в $L_{r}$ $\left[x_{n}\right]_{r} \approx \ell_{r}$ (изоморфизм). Однако, если $p<\infty$, то ввиду неравенства Хинчина [6, с. 341] $\left[r_{n}\right]_{p} \approx\left[r_{n}\right]_{q} \approx \ell_{2}\left(r_{n}-\right.$ функции Радемахера) и, следовательно, $I$ не строго сингулярен. 
В то же время, если $X$ имеет базис Шаудера, состоящий из дизъюнктных векторов, нетрудно показать, что класс DSS-операторов, определенных на $X$, совпадает с классом строго сингулярных операторов [7].

Понятие DSS-оператора оказалось важным при изучении геометрических свойств функциональных пространств. Так, например, существование операторов, не обладающих DSS-свойством, позволяет строить дополняемые подпространства с “нестандартными" проекторами на них [5], [7].

Цель настоящей работы - изучение вопроса, когда оператор тождественного вложения (далее мы всегда его обозначаем через $I$ ) одного симметричного пространства в другое обладает DSS-свойством. Условия при этом формулируются в терминах фундаментальных функций пространств.

Если $z=z(w)$ измерима на $[0,1], \mu$-мера Лебега на этом отрезке, то ее функиией распределения будем назьвать функцию $n_{z}(\tau)=\mu\{w:|z(w)|>\tau\}(\tau>0)$. Говорят, что функции $x(t)$ и $y(t)$ равноизмеримы, если $n_{x}(\tau)=n_{y}(\tau)(\tau>0)$.

Напомним, что банахово пространство $E$ измеримых функций, определенных на [0,1], назьвается симметричным (СП), если выполнены следующие условия:

1) из того, что $y \in E$ и $|x(t)| \leqslant|y(t)|$, следует $x \in E$ и $\|x\| \leqslant\|y\|$;

2 ) если $y \in E$ и функции $x(t)$ и $y(t)$ равноизмеримы, то $x \in E$ и $\|x\|=\|y\|$.

Фундаментальная функция СП $E$ определяется равенством $f_{E}(t)=\left\|\chi_{(0, t)}\right\|_{E}$, где, как обычно,

$$
\chi_{U}(t)= \begin{cases}1, & t \in U \\ 0, & t \notin U .\end{cases}
$$

Функция $f_{E}(t)$ является квазивогнутой на $(0,1][8$, с. 137$]$, т.е. она неотрицательна, возрастает, а функция $f(t) / t$ убьвает. Как известно (см., например, [8, с. 70]), такая функция эквивалентна своей наименьшей вогнутой мажоранте. Класс всех положительных возрастающих вогнутых на $(0,1]$ функций далее обозначаем через $G$.

Важным примером СП является пространство Орлича. Пусть $N(t)$ - возрастающая выпуклая функция на $[0, \infty), N(0)=0, N(\infty)=\infty$. Пространство Орлича $L_{N}$ состоит из всех измеримых на $[0,1]$ функций $x=x(t)$, для которых при некотором $u>0$

$$
\int_{T} N\left(\frac{|x(t)|}{u}\right) d \mu<\infty
$$

с нормой

$$
\|x\|=\inf \left\{u>0: \int_{T} N\left(\frac{|x(t)|}{u}\right) d \mu \leqslant 1\right\} .
$$

Непосредственное вычисление показьвает, что фундаментальная функция пространства $L_{N}$ есть $f_{N}(t)=1 / N^{-1}(1 / t)\left(N^{-1}(u)\right.$ - функция, обратная к $\left.N(u)\right)[9]$.

В работе [5] доказана следующая теорема о дизъюнктной строгой сингулярности оператора вложения пространства $L_{N}$ в пространство $L_{M}$.

Теорема. Пусть $L_{N} \subset L_{M}$. Следуюшие условия әквивалентны:

1) onepamop $I: L_{N} \rightarrow L_{M}$ - DSS-onepamop;

2) для любых $n=1,2, \ldots u \mathscr{K}>0$ существуют $1 \leqslant x_{1}<x_{2}<\cdots<x_{n} u$ $c_{1}>0, \ldots, c_{n}>0$ maкие, что

$$
\sum_{i=1}^{n} c_{i} N\left(t x_{i}\right) \geqslant \mathscr{K} \sum_{i=1}^{n} c_{i} M\left(t x_{i}\right) \quad(t \geqslant 1) .
$$


Покажем, что условие 2) этой теоремы следует из соотношения

$$
\lim _{t \rightarrow 0} \frac{f_{M}(t)}{f_{N}(t)}=0
$$

где $f_{N}$ и $f_{M}$ - фундаментальные функции пространств Орлича. Действительно, в этом случае для произвольного $0<h \leqslant 1 N^{-1}(t) \leqslant h M^{-1}(t)\left(t \geqslant t_{0}\right)$, откуда ввиду вьпуклости $N(t) M(t) \leqslant N(h t) \leqslant h N(t)$, если $t \geqslant M^{-1}\left(t_{0}\right)$. Следовательно,

$$
\lim _{t \rightarrow \infty} \frac{M(t)}{N(t)}=0
$$

и условие 2) вьполнено.

Последнее обстоятельство делает естественной постановку следующей общей задачи.

Предположим, что для функций $\varphi \in G$ и $\psi \in G$ выполнено условие

A) $\lim _{t \rightarrow 0} \frac{\psi(t)}{\varphi(t)}=0$.

Будет ли тогда оператор тождественного вложения $I: E \rightarrow F$ иметь DSS-свойство для СП $E$ и $F, E \subset F$, с фундаментальными функциями $\varphi$ и $\psi$ соответственно?

В дальнейшем будет показано, что для “классических" СП (например, пространств Лоренца и Марцинкевича) так же, как и для пространств Орлича, ответ на поставленный вопрос положителен. Более того, это верно для вложения пространства Лоренца в произвольное СП (и наоборот, произвольного СП в пространство Марцинкевича).

В общем случае, однако, это не так: в работе приведен пример двух СП $E$ и $F, E \subset F$, для фундаментальных функций которых вьполнено условие А), но оператор $I: E \rightarrow F-$ не DSS-оператор.

В то же время, с помощью фундаментальных функций пространств можно сформулировать более сильное, чем А), условие, при котором ответ на поставленный вопрос положителен для всех СП. Напомним прежде определение показателей растяжения функции.

Для положительной функции $f$, определенной на $(0,1]$, функиия растя жсения $\mathscr{M}_{f}(t)$ определяется равенством

$$
\mathscr{M}_{f}(t)=\sup \left\{\frac{f(s t)}{f(s)}: 0<s \leqslant \min \left(1, \frac{1}{t}\right)\right\} \quad(t>0)
$$

Так как $\mathscr{M}_{f}(t)$ полумультипликативна, существуют числа

$$
\gamma_{f}=\lim _{t \rightarrow 0} \frac{\ln \mathscr{M}_{f}(t)}{\ln t}, \quad \delta_{f}=\lim _{t \rightarrow \infty} \frac{\ln \mathscr{M}_{f}(t)}{\ln t},
$$

называемые нижним и верхним показателями растяжения функции $f$. Если $\varphi \in G$, то $0 \leqslant \gamma_{\varphi} \leqslant \delta_{\varphi} \leqslant 1[8$, c. 76$]$.

Для функций $\varphi$ и $\psi$ из класса $G$ введем условие

Б) $\gamma_{\psi / \varphi}>0$. 
По определению нижнего показателя растяжения легко получить, что из условия Б) следует условие А). Обратное, конечно, неверно: в качестве $\varphi$ и $\psi$ достаточно взять функции, "различающиеся" на логарифм (см. также доказательство теоремы 3).

Мы покажем, что при выполнении Б) оператор $I: E \rightarrow F$ будет DSS-оператором для произвольных СП $E$ и $F, E \subset F$, с фундаментальными функциями $\varphi$ и $\psi$ соответственно. Этот результат обобщает и одновременно уточняет аналогичную теорему для пространств Орлича из [7]. Попутно будет доказано, что условие А) необходимо и достаточно для того, чтобы оператор тождественного вложения одного пространства Лоренца в другое пространство Лоренца обладал DSS-свойством (и аналогичное утверждение для пространств Марцинкевича). Эти результаты дополняют сформулированную выше теорему для пространств Орлича из работы [5].

1. О вложениях $\Lambda(\varphi) \subset F$ и $E \subset M(\theta)$. Если функция $\varphi \in G$, то пространство Лоренца $\Lambda(\varphi)$ состоит из всех измеримых на $[0,1]$ функций $x=x(s)$, для которых

$$
\|x\|_{\Lambda(\varphi)}=\int_{0}^{1} x^{*}(s) d \varphi(s)<\infty
$$

где $x^{*}(s)$ - убьвающая непрерывная слева перестановка функции $|x(s)|[8$, c. 83]. Ясно, что фундаментальная функция пространства Лоренца $f_{\Lambda(\varphi)}(t)=\varphi(t)$.

Теорема 1. Пусть для функиий $\varphi \in G u \psi \in G$ выполнено соотношение A). Тогда если $F$ - СП на $[0,1]$ с фундаментальной функиией $\psi(t)$, то $\Lambda(\varphi) \subset F u$ оператор тохдественного вложения $I: \Lambda(\varphi) \rightarrow F$ - DSS-оператор.

ДоКАЗАТЕЛЬСТВО. Из условия А) и непрерывности при $t>0$ вогнутых функций $\varphi$ и $\psi$ следует, что для $t \in[0,1] \psi(t) \leqslant C_{1} \varphi(t)$. Поэтому ввиду определения нормы в пространстве Лоренца $\Lambda(\varphi) \subset \Lambda(\psi)$. Так как пространство Лоренца - минимальное среди СП с такой же фундаментальной функцией [7, с. 160], получаем, что $\Lambda(\varphi) \subset F$.

Предположим, что $I: \Lambda(\varphi) \rightarrow F$ не имеет DSS-свойства. Тогда существует последовательность ненулевых дизъюнктных функций $x_{n} \geqslant 0$, для которой

$$
\left\|x_{n}\right\|_{\Lambda(\varphi)} \leqslant C_{2}\left\|x_{n}\right\|_{F} \quad(n=1,2, \ldots)
$$

По условию А) для любого $0<\varepsilon<1$ существует $h>0$ такое, что при $0<t<h$

$$
\psi(t) \leqslant \varepsilon \varphi(t)
$$

Найдем $N$ такое, что при $n \geqslant N \mu\left(g_{n}\right)<h$, где $g_{n}=\left\{t \in[0,1]: x_{n}(t) \neq 0\right\}$.

В любом пространстве Лоренца на $[0,1]$ всюду плотно множество конечнозначных функций $[8$, с. 149]. Поэтому для всякого $n \geqslant N$ существует функция

$$
y_{n}(t)=\sum_{k=1}^{m_{n}} a_{k}^{n} \chi_{e_{k}^{n}}, \quad a_{k}^{n} \geqslant 0, \quad e_{1}^{n} \supset e_{2}^{n} \supset \cdots \supset e_{m_{n}}^{n}, \quad \mu\left(e_{1}^{n}\right)<h,
$$

для которой

$$
\max \left(\left\|x_{n}-y_{n}\right\|_{\Lambda(\varphi)},\left\|x_{n}-y_{n}\right\|_{\Lambda(\psi)}\right)<\varepsilon \min \left(\left\|x_{n}\right\|_{\Lambda(\varphi)},\left\|x_{n}\right\|_{\Lambda(\psi)}\right)
$$


Следовательно, $\left\|x_{n}\right\|_{\Lambda(\psi)}-\left\|y_{n}\right\|_{\Lambda(\psi)} \leqslant \varepsilon\left\|x_{n}\right\|_{\Lambda(\psi)}$ и, значит, ввиду [8, c. 160] и (2)

$$
\begin{aligned}
\left\|x_{n}\right\|_{F} & \leqslant\left\|x_{n}\right\|_{\Lambda(\psi)} \leqslant \frac{1}{1-\varepsilon}\left\|y_{n}\right\|_{\Lambda(\psi)}=\frac{1}{1-\varepsilon} \sum_{k=1}^{m_{n}} a_{k}^{n} \psi\left(\mu\left(e_{k}^{n}\right)\right) \\
& \leqslant \frac{\varepsilon}{1-\varepsilon} \sum_{k=1}^{m_{n}} a_{k}^{n} \varphi\left(\mu\left(e_{k}^{n}\right)\right)=\frac{\varepsilon}{1-\varepsilon}\left\|y_{n}\right\|_{\Lambda(\varphi)} .
\end{aligned}
$$

Из неравенства (3) следует, кроме того, что $\left\|y_{n}\right\|_{\Lambda(\varphi)} \leqslant(1+\varepsilon)\left\|x_{n}\right\|_{\Lambda(\varphi)}$. Таким образом,

$$
\left\|x_{n}\right\|_{F} \leqslant \frac{\varepsilon(1+\varepsilon)}{1-\varepsilon}\left\|x_{n}\right\|_{\Lambda(\varphi)} .
$$

Последнее противоречит неравенству (1), если выбрать $\varepsilon>0$ так, что

$$
\frac{1-\varepsilon}{\varepsilon(1+\varepsilon)}>C_{2}
$$

СледствиЕ 1. Пусть $\varphi \in G, \psi \in G, \psi(t) \leqslant C_{1} \varphi(t)(t \in(0,1])$. Следующие условия эквивалентны:

1) выполнено А);

2) onepamop $I: \Lambda(\varphi) \rightarrow \Lambda(\psi)$ - DSS-onepamop;

3) не существует последовательности дизбюнктных функиий $x_{n}$ таких, что при некотором $C_{2}>0$

$$
\left\|x_{n}\right\|_{\Lambda(\varphi)} \leqslant C_{2}\left\|x_{n}\right\|_{\Lambda(\psi)} \quad(n=1,2, \ldots) .
$$

ДокАЗАТЕЛЬСТво. Импликации 1) $\Longrightarrow 2$ ) и 2) $\Longrightarrow 3$ ) следуют из теоремы 1 и определения DSS-оператора соответственно.

Предположим, что условие А) не вьполнено, т.е.

$$
\varlimsup_{t \rightarrow 0} \frac{\psi(t)}{\varphi(t)}>0 \text {. }
$$

Тогда существует последовательность $\left\{t_{k}\right\} \subset(0,1]$ и $C_{2}>0$ такие, что $\sum_{n=1}^{\infty} t_{n} \leqslant 1$ и $\varphi\left(t_{n}\right) \leqslant C_{2} \psi\left(t_{n}\right)(n=1,2, \ldots)$. В этом случае для $x_{n}=\chi_{e_{n}}$, где $e_{n} \subset[0,1]$ не пересекаются и $\mu\left(e_{n}\right)=t_{n}$, выполнено неравенство (4). Поэтому из 3 ) вытекает 1$)$, и следствие доказано.

Пусть функция $\theta \in G$. Пространство Марцинкевича $M(\theta)$ состоит из всех измеримых на $[0,1]$ функций $x=x(s)$, для которых

$$
\|x\|_{M(\theta)}=\sup _{0<t \leqslant 1}(\theta(t))^{-1} \int_{0}^{t} x^{*}(s) d s<\infty .
$$

$\Phi$ ундаментальная функция пространства $M(\theta) f_{M(\theta)}(t)=\widetilde{\theta}(t)=t / \theta(t)$.

ТЕОрема 2. Предполохим, что для функиий $\varphi \in G$ и $\psi \in G$ выполнено условие А). Если $E-\mathrm{C \Pi} \mathrm{на}[0,1]$ с фундаментальной функиией $\varphi(t)$, то $E \subset M(\widetilde{\psi}) u$ оператор тождественного вложения $I: E \rightarrow M(\widetilde{\psi})$ - DSS-оператор. 
ДокАЗАТЕльство. Так как пространство Марцинкевича максимально среди СП с той же фундаментальной функцией [8, с. 162], имеем $E \subset M(\widetilde{\psi})$. Ввиду условия А) $\psi(t) \leqslant C_{1} \varphi(t)(t \in(0,1])$, значит по определению пространства Марцинкевича $M(\widetilde{\varphi}) \subset$ $M(\widetilde{\psi})$. Следовательно, $E \subset M(\widetilde{\psi})$.

Предположим, что $I: E \rightarrow M(\widetilde{\psi})$ не обладает DSS-свойством. Тогда, в частности, существует последовательность дизъюнктных функций $x_{n}$ такая, что

$$
\left\|x_{n}\right\|_{M(\tilde{\psi})}=1, \quad\left\|x_{n}\right\|_{M(\tilde{\varphi})} \leqslant C_{2} \quad(n=1,2, \ldots) .
$$

Найдем $t_{k} \in(0,1]$, для которых

$$
\int_{0}^{t_{k}} x_{k}^{*}(s) d s \geqslant \frac{1}{2} \widetilde{\psi}\left(t_{k}\right) \quad(k=1,2, \ldots) .
$$

Так как функции $x_{k}$ дизъюнктны, можно считать, что $t_{k} \rightarrow 0$. Поэтому

$$
\left\|x_{k}\right\|_{M(\widetilde{\varphi})} \geqslant\left(\widetilde{\varphi}\left(t_{k}\right)\right)^{-1} \int_{0}^{t_{k}} x_{k}^{*}(s) d s \geqslant \frac{\varphi\left(t_{k}\right)}{2 \psi\left(t_{k}\right)} .
$$

Ввиду А) при $k \rightarrow \infty\left\|x_{k}\right\|_{M(\widetilde{\varphi})} \rightarrow \infty$, что противоречит условию (5).

Теорема 2 доказана.

СлеДСтвиЕ 2. Пусть $\varphi \in G, \psi \in G, \psi(t) \leqslant C_{1} \varphi(t)(t \in(0,1])$. Следующие условия әквивалентны:

1) выполнено А);

2) onepamop $I: M(\widetilde{\varphi}) \rightarrow M(\widetilde{\psi})$ - DSS-onepamop;

3) не существует последовательности дизбюнктных функиий $x_{n}$ таких, что при некотором $C_{2}>0$

$$
\left\|x_{n}\right\|_{M(\widetilde{\varphi})} \leqslant C_{2}\left\|x_{n}\right\|_{M(\tilde{\psi})} \quad(n=1,2, \ldots) .
$$

Доказательство совершенно аналогично доказательству следствия 1.

СледСТВИЕ 3. Для произвольного СП $E$ на $[0,1], E \neq L_{1}$, оператор $I: E \rightarrow L_{1}-$ DSS-onepamop.

ДокАЗАТЕЛьСтво. Прежде всего, $L_{1}=M(1)$ и произвольное СП $E$ вложено в $L_{1}[8$, c. 124]. Если $f_{E}(t)=\varphi(t)$, то ввиду вогнутости $\varphi$ функция $t / \varphi(t)$ возрастает. Поэтому невьполнение условия А) эквивалентно тому, что $\varphi(t) \approx t$ (т.е. $C_{1} t \leqslant \varphi(t) \leqslant C_{2} t$ с некоторыми $C_{1}>0$ и $C_{2}>0$ ). Следовательно, $E=L_{1}$. Осталось воспользоваться теоремой 2 .

ЗАмЕЧАнИЕ 1. Иным способом утверждение последнего следствия было доказано B [10].

ЗАмечание 2. Рассуждая так же, как в следствии 3, с помощью теоремы 1 нетрудно показать, что для любого СП $E, E \neq L_{\infty}$, оператор $I: L_{\infty} \rightarrow E$ - DSS-оператор. В [11], более того, показано, что этот оператор даже строго сингулярен. Это обобщает упоминавшуюся во введении теорему Гротендика.

В следующем пункте мы докажем, что условие А), вообще говоря, не достаточно для того, чтобы оператор вложения СП с фундаментальной функцией $\varphi$ в СП с фундаментальной функцией $\psi$ обладал DSS-свойством. 
2. Пример СП $E$ и $F, E \subset F$, для фундаментальных функций которых выполнено условие A), но $I: E \rightarrow F$ - не DSS-оператор.

Теорема 3. Существуют два СП на [0,1] $E$ и $F, E \subset F$, с фундаментальными функииями $\varphi$ и н соответственно, удовлетворяющими условию А), такие, что оператор $I: E \rightarrow F$ не обладает DSS-свойством.

ДокАЗАтЕльство. В качестве СП $E$ возьмем пространство Марцинкевича $M(\widetilde{\psi})$, $\widetilde{\psi}(t)=t / \psi(t)$

$$
\psi(t)=t^{1 / 2} \log _{2}^{1 / 2} \frac{4}{t}, \quad 0<t \leqslant 1 .
$$

Непосредственно проверяется, что $\psi$ - возрастающая вогнутая функция на $(0,1], \gamma_{\psi}=$ $\delta_{\psi}=1 / 2$. Следовательно [3, с. 156],

$$
\|x\|_{M(\tilde{\psi})} \approx \sup _{0<t \leqslant 1}\left\{x^{*}(t) \psi(t)\right\}
$$

Перейдем к определению пространства $F$. Обозначим

$$
b_{k}=(k+2)^{-1 / 2} 2^{k / 2}, \quad z_{k}(t)=b_{k} \chi_{\left(0,2^{-k}\right]}(t) .
$$

Кроме того, определим последовательность номеров $n_{0}=1<n_{1}<\cdots<n_{m}<\cdots$ :

$$
n_{m+1}=\max \left\{n=1,2, \cdots: \sum_{k=n_{m}}^{n-1} \frac{1}{k+2} \leqslant 1\right\}
$$

и последовательность функций $w_{m}=w_{m}(t)$ :

$$
w_{m}(t)=\max _{n_{m} \leqslant k<n_{m+1}} z_{k}(t), \quad m=0,1, \ldots
$$

Так как $\left\{b_{k}\right\}$ возрастает, ввиду $(7)$ норма функций $w_{m}$ в $L_{2}$ удовлетворяет неравенствам

$$
\begin{gathered}
\left\|w_{m}\right\|_{2}^{2} \geqslant \sum_{k=n_{m}}^{n_{m+1}-1} b_{k}^{2} 2^{-k-1}=\frac{1}{2} \sum_{k=n_{m}}^{n_{m+1}-1} \frac{1}{k+2} \geqslant \frac{1}{4} \\
\left\|w_{m}\right\|_{2}^{2} \leqslant \sum_{k=n_{m}}^{n_{m+1}-1} b_{k}^{2} 2^{-k}=\sum_{k=n_{m}}^{n_{m+1}-1} \frac{1}{k+2} \leqslant 1 .
\end{gathered}
$$

Следовательно,

$$
\frac{1}{2} \leqslant\left\|w_{m}\right\|_{2} \leqslant 1
$$

Обозначим $\chi_{b}=b^{-1 / 2} \chi_{(0, b)}, \bar{w}_{m}=w_{m} /\left\|w_{m}\right\|_{2}$ и $V=\left\{\chi_{b}\right\}_{0<b \leqslant 1} \cup\left\{\bar{w}_{m}\right\}_{m=0}^{\infty}$. Пусть $F$ - множество всех измеримых на $[0,1]$ функций $x=x(t)$, для которых

$$
\|x\|=\sup \left\{\int_{0}^{1} x^{*}(t) v(t) d t: v \in V\right\}<\infty .
$$

Тогда $F-$ СП на $[0,1]$ как пересечение пространств Лоренца, построенных по функциям $\int_{0}^{t} v(s) d s(v \in V)$. Кроме того, из определения $F$ следует, что $\|x\|_{M\left(t^{1 / 2}\right)} \leqslant\|x\|_{F} \leqslant\|x\|_{2}$. 
Поэтому фундаментальные функции пространств $E$ и $F \quad f_{E}(t)=\psi(t)$ и $f_{F}(t)=t^{1 / 2}$ удовлетворяют условию А).

Покажем, что

$$
M(\widetilde{\psi}) \subset F .
$$

Для этого ввиду (6) достаточно показать, что функция $1 / \psi \in F$. Действительно,

$$
\int_{0}^{1} \chi_{b}(t) \frac{d t}{\psi(t)}=2 b^{-1 / 2} \int_{0}^{b} \frac{d\left(t^{1 / 2}\right)}{\log _{2}^{1 / 2}(4 / t)} \leqslant 2 \quad(0<b \leqslant 1)
$$

a

$$
\begin{aligned}
\int_{0}^{1} w_{m}(t) \frac{d t}{\psi(t)} & \leqslant \sum_{k=n_{m}}^{n_{m+1}-1} b_{k} \int_{0}^{2^{-k}} \frac{d t}{\psi(t)}=2 \sum_{k=n_{m}}^{n_{m+1}-1} b_{k} \int_{0}^{2^{-k}} \frac{d\left(t^{1 / 2}\right)}{\log _{2}^{1 / 2}(4 / t)} \\
& \leqslant 2 \sum_{k=n_{m}}^{n_{m+1}-1} \frac{1}{k+2} \leqslant 2
\end{aligned}
$$

Следовательно, ввиду (8) по определению $F$ получаем, что $\|1 / \psi\|_{F} \leqslant 4$ и вложение (9) доказано.

Обозначим далее $D_{m}=\left(2^{-n_{m+1}}, 2^{-n_{m}}\right]$,

$$
v_{m}(t)=w_{m}(t) \chi_{D_{m}}(t)=\sum_{k=n_{m}}^{n_{m+1}-1} b_{k} \chi_{\left(2^{-k-1}, 2^{-k}\right]}(t) \quad(m=0,1, \ldots) .
$$

$\Phi$ ункции $v_{m}$ дизъюнктны. Покажем, что на их линейной оболочке нормыпространств $E$ и $F$ эквивалентны.

Пусть

$$
v(t)=\sum_{m=0}^{r} a_{m} v_{m}(t)
$$

Без ограничения общности можно считать, что $a_{m} \geqslant 0$. Обозначим

$$
w(t)=\max _{0 \leqslant m \leqslant r} a_{m} w_{m}(t)
$$

Тогда $w(t)$ монотонно убывает на $(0,1]$ и $v(t) \leqslant w(t)$. Следовательно, ввиду $(6)$

$$
\|v\|_{E} \leqslant\|w\|_{E} \leqslant C \max _{0 \leqslant m \leqslant r}\left\{a_{m} \max _{n_{m} \leqslant k<n_{m+1}} b_{k} \psi\left(2^{-k}\right)\right\} .
$$

Так как $b_{k} \psi\left(2^{-k}\right)=1(k=0,1,2, \ldots)$, получаем

$$
\|v\|_{E} \leqslant C \max _{0 \leqslant m \leqslant r} a_{m} .
$$

Оценим теперь $\|v\|_{F}$ снизу. Для любого $m=0,1, \ldots, r$ ввиду (7)

$$
\begin{aligned}
\int_{0}^{1} v_{m}^{*}(t) w_{m}(t) d t & \geqslant \int_{0}^{1}\left(v_{m}^{*}(t)\right)^{2} d t=\int_{0}^{1} v_{m}^{2}(t) d t=\sum_{k=n_{m}}^{n_{m+1}-1} b_{k}^{2} 2^{-k-1} \\
& =\frac{1}{2} \sum_{k=n_{m}}^{n_{m+1}-1} \frac{1}{k+2} \geqslant \frac{1}{4}
\end{aligned}
$$


Поэтому из (8) следует, что $\left\|v_{m}\right\|_{F} \geqslant 1 / 4$. Следовательно, ввиду (10)

$$
\|v\|_{F} \geqslant \max _{0 \leqslant m \leqslant r}\left\{a_{m}\left\|v_{m}\right\|_{F}\right\} \geqslant \frac{1}{4} \max _{0 \leqslant m \leqslant r} a_{m} \geqslant \frac{\|v\|_{E}}{4 C}
$$

Вместе с вложением (9) это означает, что на линейной оболочке множества функций $v_{m}$ $(m=0,1, \ldots)$ нормы пространств $E$ и $F$ эквивалентны. Следовательно, сушествует $B>0$ такое, что для произвольных $a_{m}$

$$
B^{-1}\left\|\sum_{m=0}^{\infty} a_{m} v_{m}\right\|_{F} \leqslant\left\|\sum_{m=0}^{\infty} a_{m} v_{m}\right\|_{E} \leqslant B\left\|\sum_{m=0}^{\infty} a_{m} v_{m}\right\|_{F} .
$$

Иначе говоря, оператор тождественного вложения $I: E \rightarrow F$ не обладает DSS-свойством.

Теорема 3 доказана.

ЗАмечАниЕ 3 . Теорема 3 показывает, что соотношение А) не обеспечивает такого “зазора" между пространствами, который был бы достаточен для того, чтобы соответствующий оператор тождественного вложения обладал DSS-свойством. С другой стороны, простые примеры свидетельствуют о том, что в случае СП даже с одной и той же фундаментальной функцией этот оператор может таковьм оказаться.

Tак, например, DSS-свойством обладает оператор вложения пространства Лоренца $\Lambda\left(t^{1 / p}\right)$ в пространство $L_{p}(1<p<\infty)$. Действительно, нетрудно показать, что из любой последовательности нормированных дизъюнктных функций в пространстве Лоренца можно выделить подпоследовательность, эквивалентную стандартному базису в $\ell_{1}$. В то же время, любая такая последовательность функций в $L_{p}$ эквивалентна стандартному базису в $\ell_{p}$.

В заключительном пункте мы покажем, что в отличие от А) условие Б) достаточно для того, чтобы оператор $I: E \rightarrow F$ был DSS-оператором, если $E$ и $F$ - произвольные СП с фундаментальными функциями $\varphi$ и $\psi$ соответственно. Попутно будет установлено важное и само по себе DSS-свойство оператора вложения пространства Марцинкевича в пространство Лоренща.

\section{3. Достаточность условия Б) для того, чтобы оператор $I: E \rightarrow F$ обладал DSS-свойством.}

ТЕорема 4. Пусть функиии $\varphi \in G, \psi \in G, \delta_{\varphi}<1, u$ для этих функиий вьполнено условие А). Если $M(\widetilde{\varphi}) \subset \Lambda(\psi)$, то оператор $I: M(\widetilde{\varphi}) \rightarrow \Lambda(\psi)$ обладает DSS-свойством.

Докажем сначала вспомогательное утверждение.

ЛЕмма. В условиях теоремы 4 существует функиия $\rho \in G$, для которой
1) $\lim _{t \rightarrow 0} \frac{\rho(t)}{\varphi(t)}=0$;
2) $M(\widetilde{\rho}) \subset \Lambda(\psi)$. 
ДокАЗАТЕЛЬСТво. Так как $\delta_{\varphi}<1$, ввиду $[8$, с. 156$]$

$$
\|x\|_{M(\widetilde{\varphi})} \approx \sup _{0<t \leqslant 1}\left\{\varphi(t) x^{*}(t)\right\} .
$$

Поэтому вложение $M(\widetilde{\varphi}) \subset \Lambda(\psi)$ эквивалентно тому, что

$$
\int_{0}^{1} \frac{d \psi(s)}{\varphi(s)}<\infty
$$

Так как функция $\varphi$ вогнута, имеем

$$
\int_{0}^{1} \frac{d \psi(s)}{\varphi(s)} \approx \sum_{k=0}^{\infty} \frac{\psi\left(2^{-k}\right)-\psi\left(2^{-k-1}\right)}{\varphi\left(2^{-k}\right)}
$$

и, значит, (11), в свою очередь, эквивалентно условию

$$
\sum_{k=0}^{\infty} \frac{\psi\left(2^{-k}\right)-\psi\left(2^{-k-1}\right)}{\varphi\left(2^{-k}\right)}<\infty
$$

Обозначим

$$
a_{k}=\psi\left(2^{-k}\right)-\psi\left(2^{-k-1}\right), \quad S_{n}=\sum_{k=n}^{\infty} \frac{a_{k}}{\varphi\left(2^{-k}\right)} .
$$

Тогда $S_{n} \rightarrow 0$ и ввиду [12, с. 90] и (12)

$$
\sum_{k=0}^{\infty} \frac{a_{k}}{\sqrt{S_{k}} \varphi\left(2^{-k}\right)}<\infty
$$

По определению верхнего показателя растяжения найдем $u>0$ и $C>0$ такие, что $\delta_{\varphi}+u<1$ и для $t \geqslant 1$

$$
\mathscr{M}_{\varphi}(t) \leqslant C t^{\delta_{\varphi}+u / 2}
$$

Введем числовую последовательность

$$
g_{0}=S_{0}, \quad g_{k}=\max \left(S_{k}, 2^{-u} g_{k-1}\right) \quad(k=1,2, \ldots) .
$$

Пусть $g=g(t)$ - функция, линейная на отрезках $\left[2^{-k-1}, 2^{-k}\right]$, и $g\left(2^{-k}\right)=g_{k}(k=0,1, \ldots)$, $\mathrm{a} h(t)=\sqrt{g(t)} \varphi(t)$.

Так как $\left\{S_{k}\right\}$ убывает, то и $\left\{g_{k}\right\}$ убывает, а значит, функции $g(t)$ и $h(t)$ возрастают на $(0,1]$. Если $j \geqslant 0$ и $2^{-k-1}<t \leqslant 2^{-k}$, то из (15) следует

$$
\frac{g\left(2^{j} t\right)}{g(t)} \leqslant \frac{g\left(2^{j-k}\right)}{g\left(2^{-k-1}\right)}=\frac{g_{k-j}}{g_{k+1}}=\frac{g_{k+1-(j+1)}}{g_{k+1}} \leqslant 2^{(j+1) u} .
$$

Следовательно, ввиду (14)

$$
\mathscr{M}_{h}\left(2^{j}\right)=\sup _{0<t \leqslant 2^{-j}} \frac{\varphi\left(2^{j} t\right) \sqrt{g\left(2^{j} t\right)}}{\varphi(t) \sqrt{g(t)}} \leqslant C 2^{u / 2} 2^{\left(\delta_{\varphi}+u\right) j} \quad(j=0,1, \ldots) .
$$


Так как $\delta_{\varphi}+u<1$, отсюда $\delta_{h}<1$. Поэтому ввиду [8, с. 78$]$ функция $h(t)$ эквивалентна своей наименьшей вогнутой мажоранте, которую мы обозначим через $\rho(t)$.

Функция $\rho \in G, \rho\left(2^{-k}\right) \approx h\left(2^{-k}\right)=\sqrt{g_{k}} \varphi\left(2^{-k}\right)(k=0,1,2, \ldots)$, а так как $g_{k} \geqslant S_{k}$, из (13) следует

$$
\sum_{k=0}^{\infty} \frac{a_{k}}{\rho\left(2^{-k}\right)} \leqslant C_{1} \sum_{k=0}^{\infty} \frac{a_{k}}{\sqrt{S_{k}} \varphi\left(2^{-k}\right)}<\infty .
$$

Но последнее так же, как и для функции $\varphi$ (см. (11) и (12)), эквивалентно вложению $M(\widetilde{\rho}) \subset \Lambda(\psi)$.

Наконец, из соотношения (15) получаем, что

$$
\lim _{t \rightarrow 0} \frac{\rho(t)}{\varphi(t)} \leqslant C_{1} \lim _{t \rightarrow 0} \sqrt{g(t)}=\lim _{k \rightarrow \infty} \sqrt{g_{k}}=0 .
$$

Лемма доказана.

ДОКАЗАТЕЛЬСТВо ТЕОРЕМЫ 4. По лемме найдем функцию $\rho \in G$ такую, что

$$
M(\widetilde{\varphi}) \subset M(\widetilde{\rho}) \subset \Lambda(\psi) \quad \text { и } \quad \lim _{t \rightarrow 0} \frac{\rho(t)}{\varphi(t)}=0 .
$$

Тогда ввиду следствия 2 оператор $I: M(\widetilde{\varphi}) \rightarrow M(\widetilde{\rho})$ имеет DSS-свойство; тем более, он обладает этим свойством, если его рассматривать как оператор из $M(\widetilde{\varphi})$ в $\Lambda(\psi)$.

Теорема доказана.

Последний результат позволяет доказать достаточность условия Б) для того, чтобы оператор тождественного вложения из СП $E$ в СП $F$ с фундаментальными функциями $\varphi$ и $\psi$ соответственно обладал DSS-свойством.

Tеорема 5. Пусть для функиий $\varphi \in G u \psi \in G$ выполнено условие Б). Если $Е$ и $F$ - СП с фундаментальными функииями $\varphi$ и $\psi$ соответственно, то $E \subset F u$ $I: E \rightarrow F-D S S$-onepamop.

ДоКАЗАТЕЛЬСтво. Проверим, что вьполнены условия теоремы 4 , т.е. $\delta_{\varphi}<1$ и

$$
M(\widetilde{\varphi}) \subset \Lambda(\psi) .
$$

Прежде всего, ввиду условия Б) существуют $u>0$ и $C>0$ такие, что для всех $0<t \leqslant 1$ и $0<s \leqslant 1$

$$
\frac{\psi(t s) \varphi(s)}{\psi(s) \varphi(t s)} \leqslant C t^{u} .
$$

Отсюда в силу вогнутости $\psi$ получаем

$$
\mathscr{M}_{\varphi}\left(\frac{1}{t}\right) \leqslant C \mathscr{M}_{\psi}\left(\frac{1}{t}\right) t^{u}=C t^{u-1}
$$

и, значит, $\delta_{\varphi} \leqslant 1-u<1$.

Далее, так как функция $x^{*}(t)$ убывает, то для $x \in M(\widetilde{\varphi})$

$$
x^{*}(t) \leqslant \frac{\|x\|_{M(\tilde{\varphi})}}{\varphi(t)}, \quad 0<t \leqslant 1 .
$$


Поэтому для доказательства (16) достаточно проверить, что $1 / \varphi \in \Lambda(\psi)$. Из (17) следует, что $\psi(t) / \varphi(t) \leqslant C_{1} t^{u}$. Поэтому $\psi(t) \rightarrow 0$ при $t \rightarrow 0$ и

$$
\left\|\frac{1}{\varphi}\right\|_{\Lambda(\psi)}=\int_{0}^{1} \frac{\psi^{\prime}(t)}{\varphi(t)} d t \leqslant \int_{0}^{1} \frac{\psi(t)}{\varphi(t)} \frac{d t}{t} \leqslant C_{1} u^{-1}<\infty .
$$

Итак, вложение (16) доказано. Ввиду уже упоминавшейся экстремальности пространств Лоренца и Марцинкевича в классе СП с одинаковой фундаментальной функцией [8] получаем $E \subset M(\widetilde{\varphi}) \subset \Lambda(\psi) \subset F$. Поэтому $I: E \rightarrow F$ обладает DSS-свойством, так как аналогичное свойство он имеет по теореме 4 как оператор из $M(\widetilde{\varphi})$ в $\Lambda(\psi)$.

Теорема 5 доказана.

ЗАмЕчАнИЕ 4. Для пространств Орлича и при несколько более ограничительном чем Б) условии на функции $\varphi$ и $\psi: \delta_{\varphi}<\gamma_{\psi}$, последнее утверждение было получено в работе [7].

В заключение автор выражает благодарность С. Я. Новикову за полезные обсуждения.

\section{СПИСОК ЦИТИРОВАННОЙ ЛИТЕРАТУРЫ}

[1] Пич А. Операторные идеалы. М.: Мир, 1982.

[2] Grothendieck A. Sur certains sous-espaces vectoriels de $L^{p} / /$ Canad. J. Math. 1954. V. 6. P. $158-160$.

[3] Рудин У. Функциональный анализ. М.: Мир, 1975.

[4] Rodin V.A., Semenov E. M. Rademacher series in symmetric spaces // Anal. Math. 1975. V. 1. № 3. P. 207-222.

[5] Hernandez F. L., Rodrigues-Salinas B. On $\ell^{p}$-complemented copies in Orlicz spaces. 2 // Israel J. Math. 1989. V. 68. P. 27-55.

[6] Зигмунд А. Тригонометрические ряды. Т. 2. М.: Мир, 1965.

[7] Hernandez F. L. Disjointly strictly-singular operators // Proc. 19th Winter School on Analysis (Srni). Acta Univ. Carolin. Math. Phys. 1990. V. 31. P. 35-49.

[8] Крейн С. Г., Петунин Ю.И., Семёнов Е. М. Интерполяция линейных операторов. М.: Наука, 1978.

[9] Красносельский М. А., Рутицкий Я. Б. Выпуклые функции и пространства Орлича. М.: Физматгиз, 1958.

[10] Новиков С.Я. О числовой характеристике подпространства симметричного пространства // Исследования по теории функций многих вещественных переменных. Ярославль, 1980. C. $140-148$.

[11] Novikov S. Ya. Boundary spaces for inclusion map between rearrangement invariant spaces // Proc. 3rd Conf. Function Spaces (Poznan). Collect. Math. 1993. V. 44. P. 211-215.

[12] Рудин У. Основы математического анализа. М.: Мир, 1976.

Самарский государственный университет

E-mail: astashkin@univer.samara.su

Поступило 18.04 .96 